\title{
Estructura dimensional de una escala de actitudes hacia la estadística en estudiantes universitarios de segundo y tercer semestre de la facultad de Psicología de la Universidad Piloto de Colombia ${ }^{3}$
}

\author{
Vivian Andrea Bustos Velasco \\ Especialista en Estadística \\ Universidad Piloto de Colombia \\ Bogotá, Colombia \\ Correo electrónico: vivianbuve@hotmail.com \\ Heivar Yesid Rodríguez Pinzón \\ Magister en Ciencias Económicas \\ Universidad Manuela Beltrán \\ Bogotá, Colombia

\section{Resumen}

Objetivo: analizar la estructura dimensional de la escala de actitudes hacia la estadística de Auzmendi (1992). Método: se desarrolló como investigación descriptiva, se aplicó a 109 estudiantes de segundo y tercer semestre de Psicología Universidad Piloto de Colombia, los cuales fueron seleccionados por censo. Resultados: se realizó una solución factorial basada en cuatro dimensiones y quince ítems, con capacidad para explicar el $63.29 \%$ de la varianza del instrumento y con una fiabilidad alfa de Cronbach igual a .846. Conclusión: Se concluye que no son estimables las estructuras dimensionales propuestas por Auzmendi. 


\section{Dimensional structure of a scale of attitudes towards statistics on university students in second and third semester of the Faculty of Psychology of the Pillot University of Colombia.}

Abstract

Objective: to analyze the dimensional structure of Auzmendi's Scale of Attitudes toward Statistics (1992). Method: it was developed and applied as a descriptive research to 109 students of second and third semester of Psychology at Piloto University of Colombia selected by census. Results: a factorial solution was performed based on four dimensions and fifteen items, with capacity to explain the $63.29 \%$ of the instrument variance and with a reliability Cronbach's Alpha coefficient equal to .846 . Conclusion: dimensional structures proposed by Auzmendi are not estimable.

Keywords

\section{Estrutura tridimensional de uma escala de atitudes em relação a estatísticas sobre os estudantes universitários em segundo e terceiro semestre da Faculdade de Psicologia da Universidade Pilloto da Colômbia}

\section{Resumo}

Objetivo: analisar a estrutura dimensional da escala de atitudes à estatística de Auzmendi (1992). Método: se desenvolveu como investigação descritiva, se aplicou a 109 estudantes de segundo e terceiro semestre de Psicologia Universidade Piloto de Colômbia, os quais foram selecionados por censo. Resultados: se realizou uma solução fatorial baseada em quatro dimensões e quinze itens, com capacidade para explicar $63.29 \%$ da variância do instrumento e com uma confiança alfa de Cronbach igual a .846. Conclusão: Se conclui que não são estimáveis as estruturas dimensionais propostas por Auzmendi.

Palavras chave análise fatorial, escala, estatística, medição de atitude, validação. 


\section{ntroducción}

Desde el Ministerio de Educación, a partir del año 2006 se crean estándares básicos de competencia donde se menciona el pensamiento aleatorio y los sistemas de datos; este tipo de pensamiento ayuda a tomar decisiones en situaciones de incertidumbre, de azar, de riesgo o de ambigüedad por falta de información confiable, en las que no es posible predecir con seguridad lo que va a pasar, lo cual hace que la estadística sea una materia básica asignada a cualquier tipo de estudio ya sea de tipo cualitativo o cuantitativo.

Por lo anterior, se hace necesario validar un instrumento que mida la actitud del estudiante frente a la estadística, sabiendo que las actitudes son factores indispensables a la hora de juzgar su rechazo o aceptación por un nuevo aprendizaje (Cazau, 2003). La actitud es un factor predominante para adquirir un nuevo conocimiento puesto que de éste depende la orientación al éxito, fracaso, sobresfuerzo y resignación; la atribución de logro que refiere a casualidad, sí mismo, externa; y la actitud ante la tarea que es expectativa, dificultad, valor (Aliaga \& Pecho, 2000).

La actitud también ha sido definida como un estado de la disposición nerviosa y mental, puesto que da respuesta a partir de las vivencias. Por lo tanto, la actitud es una motivación social antes que una motivación biológica ya que, a partir de la experiencia, las personas adquieren una cierta predisposición que les permite responder ante los estímulos del momento (Eagly \& Chaiken, 1993).
La psicología social se encarga de estudiar las actitudes de los seres humanos para predecir posibles conductas. Cuando se observan las actitudes de un individuo, es posible pronosticar su modo de accionar (Bazán \& Aparicio, 2006). Las actitudes cumplen con diversas funciones en la vida social. Puede darse el caso de alguien que adopta una actitud defensiva y así mismo se predisponga ante la adquisición de un nuevo aprendizaje. La actitud también puede orientarse a la adaptación, en un intento por minimizar los conflictos (Gil, 1999).

La propuesta de encuesta de Auzmendi (1992) selecciona 5 factores donde muestra el agrado y confianza, la ansiedad ante la estadística, la importancia de la estadística, el interés por la estadística y la motivación mostrando factores importantes para la actitud del estudiante frente a la estadística, lo cual hace que se vuelva indispensable tomar esta prueba como base de aplicación para observar la reducción de factores según los datos arrojados por los estudiantes de la Universidad Piloto de Colombia.

El método usado para la validación del constructo fue un análisis factorial, ya que éste permite hacer un estudio donde se revisa gran cantidad de variables en una base de datos obteniendo a partir de este análisis factorial una reducción de variables. El análisis factorial exploratorio es conocido como una técnica de la estadística de interdependencia que se caracteriza por su versatilidad (García, Gil, \& Rodríguez, 2000), donde su propósito principal es tratar de estructurar internamente entre sus variables de análisis, a partir de estructuras de correlación entre ellas; en otras palabras, busca agrupar variables 
(más conocido como factores) que están altamente correlacionadas entre sí.

Primero, se presenta la Medida de Adecuación de la Muestra KMO propuesta por Kaiser, Meyer \& Olkin (1997), los cuales presentan esta medida como una forma de evaluar la prueba. Esta medida está dada por el coeficiente de correlación parcial entre las variables Xi y Xj eliminando la influencia del resto de las variables. E índice KMO es un índice que toma valores entre 0 y 1 y que se utiliza para comparar las magnitudes de los coeficientes de correlación observados con las magnitudes de los coeficientes de correlación parcial de forma que, cuanto más pequeño sea su valor, mayor es el valor de los coeficientes de correlación parciales y, por lo tanto, menos deseable es realizar un Análisis Factorial. Kaise, Meyer \& Olkin (1997) aconsejan que si $\mathrm{KMO} \geq .75$ la idea de realizar un análisis factorial es buena, si .75 > $\mathrm{KMO} \geq .5$ la idea es aceptable y si $\mathrm{KMO}<$ 0.5 es inaceptable (Gorsuch, 1983).

Segundo, se presenta el Método de Componentes Principales, el cual consiste en estimar las puntuaciones factoriales mediante las puntuaciones tipificadas de las $\mathrm{k}$ primeras componentes principales y la matriz de cargas factoriales mediante las correlaciones de las variables originales con éstos componentes. Este método tiene la ventaja de que siempre proporciona una solución. Tiene el inconveniente, sin embargo, de que al no estar basado en el modelo de Análisis Factorial puede llevar a estimadores muy sesgados de la matriz de cargas factoriales, particularmente, si existen variables con comunalidades bajas (García, Gil, \& Rodríguez, 2000).

La correlación entre dos variables elevada al cuadrado se conoce como su total de varianza compartida. La varianza total de cualquier variable puede ser dividida en tres partes, así: a la cantidad de varianza que es compartida con las demás variables en análisis se le conoce como varianza común o comunalidad; a la cantidad de varianza que sólo depende de la variable y no puede explicarse por medio de otras se le conoce como varianza específica o unicidad, y, finalmente, a la cantidad de varianza que se debe al error aleatorio se le conoce como error de varianza. La varianza total corresponde a la suma de estos tres componentes (Hair, Black, \& Babin, 2009).

Tercero, se realizó la interpretación de los factores, la importancia radica en gran parte de la experiencia y del conocimiento que el investigador tenga del problema o caso. La estimación de la matriz de factores, que contiene las ponderaciones de cada variable y la rotación de factores; este último con el objetivo de redistribuir la varianza de las variables originales en los factores para lograr una mejor interpretación de los resultados.

El juzgamiento de la significancia de los factores, luego de la rotación de éstos, se hace necesario para la evaluación de las ponderaciones en cada uno de ellos, con el fin de identificar más fácil el grado de aporte de cada una de las variables en los nuevos factores. Para este paso es necesario evaluar en términos estadísticos, cuáles variables son las más importantes e identificar aquellas que no aportan al análisis y pueden ser eliminadas. Para realizar la evaluación estadística se recurre a la significancia de las ponderaciones (Feigl \& Scriven, 1967).

Por último, se aplicó el método de consistencia interna basado en el alfa de 
Cronbach que permite estimar la fiabilidad de un instrumento de medida a través de un conjunto de ítems que se espera que midan el mismo constructo o dimensión teórica (Clemente, 1992). La validez de un instrumento se refiere al grado en que el instrumento mide aquello que pretende medir, y la fiabilidad de la consistencia interna del instrumento se puede estimar con el alfa de Cronbach. La medida de la fiabilidad mediante el alfa de Cronbach asume que los ítems (medidos en escala tipo Likert) miden un mismo constructo y que están altamente correlacionados. Cuanto más cerca se encuentre el valor del alfa a 1 mayor es la consistencia interna de los ítems analizados. La fiabilidad de la escala debe obtenerse siempre con los datos de cada muestra para garantizar la medida fiable del constructo en la muestra concreta de investigación. Como criterio general, sugieren las recomendaciones siguientes para evaluar los coeficientes de alfa de Cronbach: Coeficiente alfa mayor a .9 es excelente, el Coeficiente alfa mayor a .8 es bueno, el Coeficiente alfa mayor a .7 es aceptable, el Coeficiente alfa mayor a .6 es cuestionable, y si el Coeficiente alfa .5 es pobre (Cronbach, 1951).

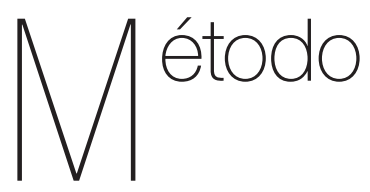

\section{Diseño}

La presente investigación se enmarca en el tipo descriptivo, ya que pretende hallar evidencias de validez en las actitudes de los estudiantes de la Universidad Piloto de Colombia frente a la estadística.

\section{Participantes}

Se seleccionaron los 109 estudiantes que conforman el segundo y tercer semestre de psicología en la Universidad Piloto de Colombia y que están cursando actualmente la materia de estadística. El 80\% pertenece al sexo femenino, el resto son del sexo masculino. Dada la forma como se seleccionó la población, se puede afirmar que es una investigación de tipo transversal, ya que en una sola ocasión se tendrá una interacción con el grupo de personas.

\section{Instrumento}

Se utilizó el cuestionario sugerido por Auzmendi (1992), el cual presenta 25 ítems que corresponden a 5 factores, el primer factor es la utilidad, la cual habla de la relación en términos de valor subjetivo que una persona le otorga a la estadística; está compuesto por los ítems 1 , $6,11,16$ y 21 . El segundo factor se refiere a la ansiedad, que muestra el sentimiento de ansiedad o temor ante la estadística; está compuesto por los ítems 2, 7, 12, 17 y 22. El tercer factor se enfoca en la confianza, el cual habla de la relación entre la confianza y seguridad que una persona muestra al trabajar con la estadística, está compuesto por los ítems $3,8,13,18$ y 23 , el cuarto factor es el agrado, en el cual se muestra el agrado o la satisfacción que provoca hacer uso de la estadística, está compuesto por los ítems 4, 9, 14, 19 y 24 y por último está el factor motivación, en este se presenta la motivación del estudiante hacia el uso y estudio de la estadística, está conformado por los ítems: 5, 10, 15, 20 y 25. 
Estos 5 factores básicos explican el 63.293\% de la varianza en nuestro caso; además, Auzmendi (1992) reporta niveles altos de consistencia interna para la escala total (Alfa de Cronbach $=.90$ ). Para nuestro estudio el Alfa de Cronbach fue de .846 .

\section{Diseño estadístico}

Con la información recogida en la aplicación del instrumento se elaboró una base de datos en el programa SPSS Statistics 22.0. El análisis permitió obtener en primer lugar los estadísticos descriptivos de cada uno de los ítems, factores y la prueba. Se realizó un análisis factorial donde se resumió la información siendo el número de factores menor que el número de variables.

Para evaluar la pertinencia del análisis factorial se estimó el estadístico de adecuación de la muestra de KaiserMeyer-Olkin (KMO). Se evaluó también, la consistencia interna de la escala y la fiabilidad de cada factor mediante el cálculo del coeficiente Alpha de Cronbach.

\section{Procedimiento}

En la primera fase el grupo investigador se dirigió a la Universidad Piloto de Colombia en Bogotá, donde se habló con la decana para autorizar la aplicación de la encuesta a los estudiantes. En la segunda fase se realizó la aplicación del instrumento en tres sesiones, puesto que son clases y horarios diferentes en los cuales se podría tener a la población de estudio; en cada grupo se dio la instrucción general de cómo se debería responder el instrumento, si existía alguna inquietud el instructor se dirigía al estudiante y se daba una solución pertinente a su pregunta, asegurando la confidencialidad de sus respuestas. En la última fase se recolectó la información, se clasificó en tablas de registro para realizar un análisis factorial, para así poder dar respuesta al objetivo planteado.

Como aspectos éticos se solicitó un consentimiento informado a los estudiantes donde se le muestra al estudiante el tipo de investigación y el uso de los datos, donde ellos firmaron su aceptación y respondieron la encuesta.

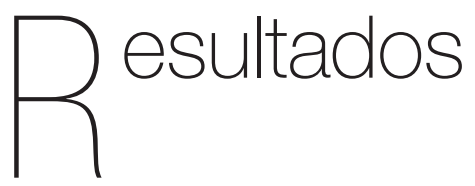

Después de realizar un análisis factorial de componentes principales y rotación varimax, se empezó reduciendo ítems dado que la fiabilidad de la prueba era muy baja y la cantidad de factores por los cuales se podía explicar eran superiores a los de la prueba de Auzmendi (1992), por lo que se quitaron los ítems 15, 8, 3, 11, 24, 6, 9, 4, 19, 23, los cuales se eliminaron dado que el Alfa de Cronbach aumentaba sin la presencia de estos ítems y estará más cercano al Alfa de Cronbach propuesto de .9.

En la Tabla 1 se muestra que con un $(\mathrm{KMO})=.793$, se puede trabajar por análisis factorial, dado que las correlaciones entre las 15 variables pueden ser explicadas por otras variables. Prueba 
de esfericidad de Barlett: $\chi 2=565.110$; g.l. $=105 ; p<.000$.

Tabla 1.

KMO y prueba de Bartlett, fuerza de la explicación de variables

\begin{tabular}{crc}
\hline \multicolumn{2}{c}{$\begin{array}{c}\text { Medida de adecuación muestral } \\
\text { de Kaiser-Meyer-Olkin. }\end{array}$} & .793 \\
\hline Chi-cuadrado aproximado & & 565.110 \\
Prueba de esfericidad de Bartlett & Gl & 105 \\
& Sig. & .000 \\
\hline
\end{tabular}

En la Tabla 2 aparecen las comunalidades asignadas inicialmente en las variables (inicial) y las comunalidades reproducidas por la solución factorial (extracción). Como se observa en la Tabla 2 las variables que peor se pueden explicar a partir de sus varianzas son los ítems 1 , 5, 7, 13, 16 con un 59.7\%, 56.8\%, 56.0\%, $59.5 \%$ y $57.5 \%$ respectivamente.

Para llegar a una solución factorial se realizó el método de componentes principales. Éste método de extracción, que es el que actúa por defecto, asume que es posible explicar el $100 \%$ de la varianza
Tabla 2.

Comunalidades, solución factorial

\begin{tabular}{ccc}
\hline & Inicial & Extracción \\
\hline Item1 & 1 & .597 \\
Item2 & 1 & .655 \\
Item5 & 1 & .568 \\
Item10 & 1 & .56 \\
Item12 & 1 & .694 \\
Item13 & 1 & .64 \\
Item14 & 1 & .595 \\
Item16 & 1 & .642 \\
Item17 & 1 & .575 \\
Item18 & 1 & .691 \\
Item20 & 1 & .623 \\
Item21 & 1 & .653 \\
Item22 & 1 & .728 \\
Item25 & 1 & .654 \\
\hline
\end{tabular}

observada. En la Tabla 3 se observa que se pueden extraer 4 factores que consiguen explicar el $63.3 \%$ de la varianza de los datos originales.

Tabla 3.

Varianza Total Explicada

\begin{tabular}{|c|c|c|c|c|c|c|c|c|c|}
\hline \multirow[t]{2}{*}{ Comp } & \multicolumn{3}{|c|}{ Autovalores iniciales } & \multicolumn{2}{|c|}{$\begin{array}{l}\text { Sumas de las } \\
\text { saturaciones al } \\
\text { cuadrado de la } \\
\text { extracción }\end{array}$} & \multirow{2}{*}{$\begin{array}{c}\begin{array}{c}\text { Sumas de las } \\
\text { saturaciones } \\
\text { al cuadrado de } \\
\text { la extracción }\end{array} \\
\% \text { acumulado }\end{array}$} & \multicolumn{2}{|c|}{$\begin{array}{l}\text { Suma de las } \\
\text { saturaciones } \\
\text { al cuadrado de } \\
\text { la rotación }\end{array}$} & \multirow[b]{2}{*}{$\begin{array}{c}\% \\
\text { acumulado }\end{array}$} \\
\hline & Total & $\begin{array}{c}\% \text { de la } \\
\text { varianza }\end{array}$ & $\begin{array}{c}\% \\
\text { acumulado }\end{array}$ & Total & $\begin{array}{c}\% \text { de la } \\
\text { varianza }\end{array}$ & & Total & $\begin{array}{c}\% \text { de la } \\
\text { varianza }\end{array}$ & \\
\hline 1 & 4.873 & 32.484 & 32.484 & 4.873 & 32.484 & 32.484 & 2.448 & 16.319 & 16.319 \\
\hline 2 & 1.785 & 1.900 & 44.384 & 1.785 & 11.900 & 44.384 & 2.401 & 16.006 & 32.324 \\
\hline 3 & 1.498 & 9.990 & 54.374 & 1.498 & 9.990 & 54.374 & 2.354 & 15.694 & 48.018 \\
\hline 4 & 1.338 & 8.919 & 63.293 & 1.338 & 8.919 & 63.293 & 2.291 & 15.275 & 63.293 \\
\hline 5 & .795 & 5.301 & 68.594 & & & & & & \\
\hline 6 & .764 & 5.094 & 73.688 & & & & & & \\
\hline 7 & .673 & 4.489 & 78.177 & & & & & & \\
\hline
\end{tabular}




\begin{tabular}{|c|c|c|c|c|c|c|c|c|c|}
\hline \multirow[t]{2}{*}{ Comp } & \multicolumn{3}{|c|}{ Autovalores iniciales } & \multicolumn{2}{|c|}{$\begin{array}{l}\text { Sumas de las } \\
\text { saturaciones al } \\
\text { cuadrado de la } \\
\text { extracción }\end{array}$} & \multirow{2}{*}{$\begin{array}{c}\begin{array}{c}\text { Sumas de las } \\
\text { saturaciones } \\
\text { al cuadrado de } \\
\text { la extracción }\end{array} \\
\% \text { acumulado }\end{array}$} & \multicolumn{2}{|c|}{$\begin{array}{l}\text { Suma de las } \\
\text { saturaciones } \\
\text { al cuadrado de } \\
\text { la rotación }\end{array}$} & \multirow[b]{2}{*}{$\begin{array}{c}\% \\
\text { acumulado }\end{array}$} \\
\hline & Total & $\begin{array}{c}\% \text { de la } \\
\text { varianza }\end{array}$ & $\begin{array}{c}\% \\
\text { acumulado }\end{array}$ & Total & $\begin{array}{c}\% \text { de la } \\
\text { varianza }\end{array}$ & & Total & $\begin{array}{c}\% \text { de la } \\
\text { varianza }\end{array}$ & \\
\hline 8 & .594 & 3.962 & 82.139 & & & & & & \\
\hline 9 & .515 & 3.434 & 85.573 & & & & & & \\
\hline 10 & .486 & 3.237 & 88.810 & & & & & & \\
\hline 11 & .455 & 3.031 & 91.841 & & & & & & \\
\hline 12 & .397 & 2.647 & 94.488 & & & & & & \\
\hline 13 & .300 & 2.001 & 96.489 & & & & & & \\
\hline 14 & .283 & 1.887 & 98.377 & & & & & & \\
\hline 15 & .244 & 1.623 & 100.000 & & & & & & \\
\hline
\end{tabular}

Método de extracción: Análisis de Componentes principales.

Tabla 4.

Matriz de componentes: Método de extracción: Análisis de componentes principales.

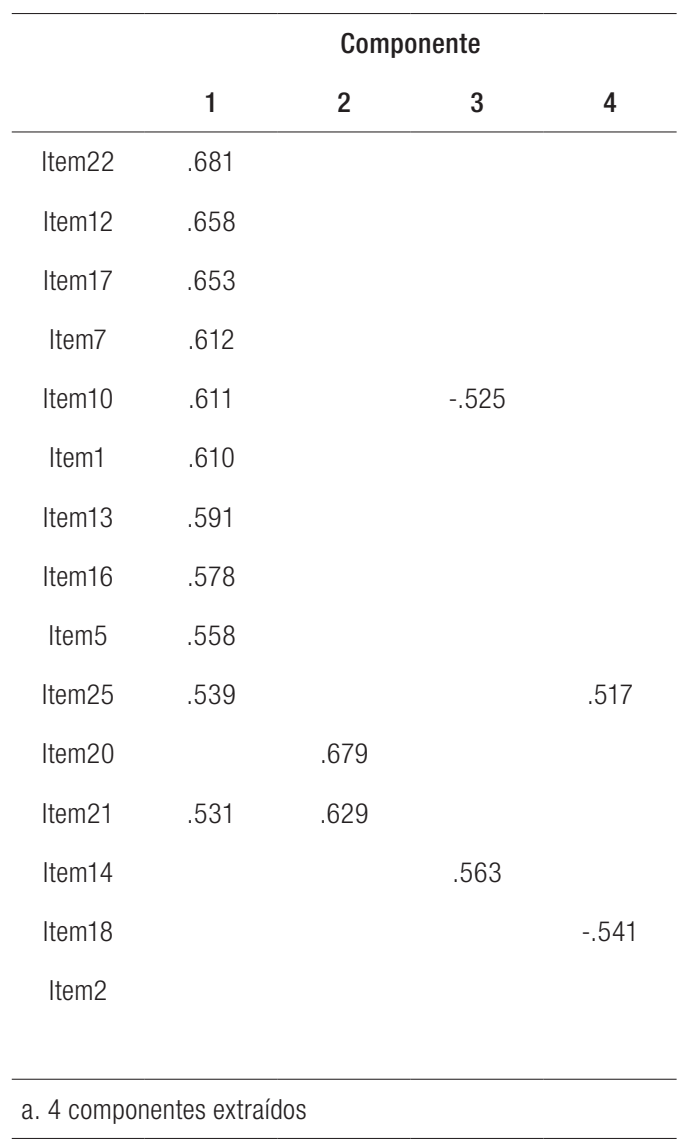

Tabla 5.

Matriz de componentes rotados, varimax

\begin{tabular}{lcccc}
\hline & \multicolumn{3}{c}{ Componente } \\
& 1 & 2 & 3 & 4 \\
\hline Item10 & .774 & & & \\
Item16 & .714 & & & \\
Item5 & .687 & & & \\
Item18 & & .774 & & \\
Item22 & & .696 & & \\
Item13 & & .667 & & \\
Item17 & & .576 & & \\
Item2 & & & .793 & .627 \\
Item12 & & & .663 & \\
Item25 & & & .612 & \\
Item7 & & & .565 & \\
Item21 & & & & \\
Item20 & & & & \\
Item1 & & & & \\
Item14 & & & & \\
\hline
\end{tabular}

Método de extracción: Análisis de componentes principales.

Método de rotación: Normalización Varimax con Kaiser. ${ }^{a}$

a. La rotación ha convergido en 6 iteraciones. 
En la Tabla 4 se compara las saturaciones relativas de cada variable en cada uno de los 4 factores. Se puede apreciar que el primer factor está constituido por los ítems 22, 12, 17, 7, 10, 1, $13,16,5$ y 25 . El factor 2 está constituido por los ítems 20 y 21; el factor 3 está constituido por el ítem 14 y el factor 4 está constituido por los ítems 18 y 2. Por tal motivo se hace la matriz de componentes rotados.

La inversa de la matriz de correlaciones se encuentra estrechamente relacionada con la matriz de anti-imagen de la Tabla 5. Teniendo en cuenta que con la inversa se puede hacer extracción, se denotan las saturaciones relativas de cada variable en cada uno de los 4 factores. Se aprecia que el primer factor está constituido por los ítems 10, 16, y 5. El factor 2 está constituido por los ítems 18, 22, 13 y 17. El factor 3 está constituido por los ítems 2, 12, 25 y 7. Y el factor 4 está constituido por los ítems 21, 20, 1 y 14.

La matriz de covarianzas anti-imagen contiene los negativos de las covarianzas parciales y la matriz de correlaciones anti-imagen contiene los coeficientes de correlación parcial cambiados de signo. Como se observa en la Tabla 6, la diagonal de la matriz de correlaciones anti-imagen tiene valores próximos a 1 y los elementos a su alrededor son pequeños, muestra que se puede trabajar por análisis factorial.

Tabla 6.

Matrices Anti-imagen

\begin{tabular}{cccccccc}
\hline & & item1 & item2 & item5 & item7 & item10 & item12 \\
\hline & Item1 & .545 & -.030 & .004 & .047 & -.120 & .001 \\
& Item2 & -.030 & .663 & .026 & -.150 & .073 & -.110 \\
& Item5 & .004 & .026 & .607 & -.015 & -.134 & -.148 \\
& Item7 & .047 & -.150 & -.015 & .548 & -.070 & -.006 \\
& Item10 & -.120 & .073 & -.134 & -.070 & .455 & -.034 \\
& Item12 & .001 & -.110 & -.148 & -.006 & -.034 & .491 \\
& Item13 & -.074 & .019 & -.078 & .053 & .004 & -.062 \\
Item14 & -.038 & -.062 & .059 & -.060 & .090 & -.120 \\
& Item16 & -.002 & .008 & -.005 & .073 & -.231 & .023 \\
& Item17 & -.062 & -.029 & .024 & -.171 & .026 & -.096 \\
& Item18 & .081 & .040 & .086 & -.032 & -.100 & .056 \\
Item20 & -.081 & .077 & .057 & -.088 & -.136 & -.020 \\
Item21 & -.164 & -.068 & -.107 & .000 & .081 & .104 \\
Item22 & -.036 & -.001 & -.007 & -.113 & -.006 & -.063 \\
Item25 & .023 & -.119 & -.094 & .027 & -.017 & -.135 \\
\hline
\end{tabular}




\begin{tabular}{|c|c|c|c|c|c|c|c|}
\hline & & item1 & item2 & item5 & item7 & item10 & item12 \\
\hline & Item1 & $.845 a$ & -.049 & .007 & .086 & -.240 & .002 \\
\hline & Item2 & -.049 & $.808 \mathrm{a}$ & .041 & -.249 & .132 & -.193 \\
\hline & Item5 & .007 & .041 & $.814 a$ & -.025 & -.255 & -.272 \\
\hline & Item7 & .086 & -.249 & -.025 & $.814 a$ & -.140 & -.012 \\
\hline & Item10 & -.240 & .132 & -.255 & -.140 & $.729 a$ & -.072 \\
\hline & Item12 & .002 & -.193 & -.272 & -.012 & -.072 & $.819 a$ \\
\hline & Item13 & -.134 & .031 & -.134 & .095 & .008 & -.118 \\
\hline \multirow[t]{16}{*}{ Correlación anti-imagen } & Item14 & -.067 & -.098 & .098 & -.105 & .173 & -.223 \\
\hline & Item16 & -.004 & .013 & -.008 & .131 & -.455 & .043 \\
\hline & Item17 & -.125 & -.054 & .046 & -.344 & .058 & -.203 \\
\hline & Item18 & .138 & .061 & .139 & -.054 & -.186 & .101 \\
\hline & Item20 & -.145 & .125 & .096 & -.158 & -.266 & -.037 \\
\hline & Item21 & -.334 & -.126 & -.206 & .000 & .179 & .222 \\
\hline & Item22 & -.068 & -.002 & -.012 & -.210 & -.013 & -.125 \\
\hline & Item25 & .040 & -.187 & -.155 & .046 & -.033 & -.247 \\
\hline & & item13 & item14 & item16 & item17 & item18 & item20 \\
\hline & Item1 & -.074 & -.038 & -.002 & -.062 & .081 & -.081 \\
\hline & Item2 & .019 & -.062 & .008 & -.029 & .040 & .077 \\
\hline & Item5 & -.078 & .059 & -.005 & .024 & .086 & .057 \\
\hline & Item7 & .053 & -.060 & .073 & -.171 & -.032 & -.088 \\
\hline & Item10 & .004 & .090 & -.231 & .026 & -.100 & -.136 \\
\hline & Item12 & -.062 & -.120 & .023 & -.096 & .056 & -.020 \\
\hline & Item13 & .560 & .074 & -.026 & -.131 & -.165 & -.023 \\
\hline \multirow[t]{15}{*}{ Covarianza anti-imagen } & Item14 & .074 & .596 & -.051 & .055 & -.120 & -.123 \\
\hline & Item16 & -.026 & -.051 & .568 & -.099 & .024 & .059 \\
\hline & Item17 & -.131 & .055 & -.099 & .453 & -.035 & .060 \\
\hline & Item18 & -.165 & -.120 & .024 & -.035 & .631 & .069 \\
\hline & Item20 & -.023 & -.123 & .059 & .060 & .069 & .571 \\
\hline & Item21 & -.094 & -.113 & -.043 & .072 & -.065 & -.167 \\
\hline & Item22 & -.064 & -.096 & -.020 & -.071 & -.141 & .043 \\
\hline & Item25 & .150 & .050 & -.094 & -.066 & -.027 & -.004 \\
\hline & Item1 & $-.134 a$ & -.067 & -.004 & -.125 & .138 & -.145 \\
\hline & Item2 & .031 & $-.098 \mathrm{a}$ & .013 & -.054 & .061 & .125 \\
\hline & Item5 & -.134 & .098 & $-.008 a$ & .046 & .139 & .096 \\
\hline & Item7 & .095 & -.105 & .131 & $-.344 a$ & -.054 & -.158 \\
\hline & Item10 & .008 & .173 & -.455 & .058 & $-.186 a$ & -.266 \\
\hline & Item12 & -.118 & -.223 & .043 & -.203 & .101 & $-.037 \mathrm{a}$ \\
\hline & Item13 & .791 & .128 & -.047 & -.260 & -.277 & -.041 \\
\hline \multirow[t]{8}{*}{ Correlación anti-imagen } & Item14 & .128 & .758 & -.087 & .106 & -.196 & -.210 \\
\hline & Item16 & -.047 & -.087 & .796 & -.194 & .040 & .104 \\
\hline & Item17 & -.260 & .106 & -.194 & .815 & -.066 & .119 \\
\hline & Item18 & -.277 & -.196 & .040 & -.066 & .758 & .114 \\
\hline & Item20 & -.041 & -.210 & .104 & .119 & .114 & .721 \\
\hline & Item21 & -.189 & -.219 & -.085 & .161 & -.123 & -.332 \\
\hline & Item22 & -.117 & -.171 & -.037 & -.145 & -.244 & .078 \\
\hline & Item25 & .257 & .083 & -.161 & -.126 & -.043 & -.007 \\
\hline
\end{tabular}




\begin{tabular}{|c|c|c|c|c|}
\hline & & item21 & item22 & item25 \\
\hline & Item1 & -.164 & -.036 & .023 \\
\hline & Item2 & -.068 & -.001 & -.119 \\
\hline & Item5 & -.107 & -.007 & -.094 \\
\hline & Item7 & .000 & -.113 & .027 \\
\hline & Item10 & .081 & -.006 & -.017 \\
\hline & Item12 & .104 & -.063 & -.135 \\
\hline & Item13 & -.094 & -.064 & .150 \\
\hline \multirow[t]{15}{*}{ Covarianza anti-imagen } & Item14 & -.113 & -.096 & .050 \\
\hline & Item16 & -.043 & -.020 & -.094 \\
\hline & Item17 & .072 & -.071 & -.066 \\
\hline & Item18 & -.065 & -.141 & -.027 \\
\hline & Item20 & -.167 & .043 & -.004 \\
\hline & Item21 & .445 & .006 & -.117 \\
\hline & Item22 & .006 & .528 & .001 \\
\hline & Item25 & -.117 & .001 & .606 \\
\hline & Item1 & $-.334 a$ & -.068 & .040 \\
\hline & Item2 & -.126 & $-.002 a$ & -.187 \\
\hline & Item5 & -.206 & -.012 & $-.155 a$ \\
\hline & Item7 & .000 & -.210 & .046 \\
\hline & Item10 & .179 & -.013 & -.033 \\
\hline & Item12 & .222 & -.125 & -.247 \\
\hline & Item13 & -.189 & -.117 & .257 \\
\hline \multirow[t]{8}{*}{ Correlación anti-imagen } & Item14 & -.219 & -.171 & .083 \\
\hline & Item16 & -.085 & -.037 & -.161 \\
\hline & Item17 & .161 & -.145 & -.126 \\
\hline & Item18 & -.123 & -.244 & -.043 \\
\hline & Item20 & -.332 & .078 & -.007 \\
\hline & Item21 & .703 & .012 & -.226 \\
\hline & Item22 & .012 & .897 & .001 \\
\hline & Item25 & -.226 & .001 & .790 \\
\hline
\end{tabular}

a. Medida de adecuación muestral

En Tabla 7 se evidencia la minimi- saturaciones altas en cada factor, lo zación del número de factores nece- cual simplifica la interpretación de los sarios, es decir, 4 factores que tienen factores.

Tabla 7.

Matriz de transformación de las componentes

\begin{tabular}{ccccc}
\hline Componente & $\mathbf{1}$ & $\mathbf{2}$ & $\mathbf{3}$ & $\mathbf{4}$ \\
\hline 1 & .530 & .537 & .504 & .419 \\
2 & .029 & -.236 & -.481 & .844 \\
3 & -.847 & .335 & .293 & .290 \\
4 & -.008 & -.737 & .655 & .167 \\
\hline
\end{tabular}

Método de extracción: Análisis de componentes principales.

Método de rotación: Normalización Varimax con Kaiser. 
Se observa en la Tabla 8 que no se ha excluido ningún dato y el $100 \%$ de los elementos fueron tenidos en cuenta en el estudio.

Tabla 8.

Resumen del procesamiento de los casos

\begin{tabular}{cccc}
\hline & & N & $\%$ \\
\hline \multirow{2}{*}{ Casos } & Válidos & 109 & 100.0 \\
\hline & Excluidos $^{\mathrm{a}}$ & 0 & .0 \\
\hline & Total & 109 & 100.0 \\
\hline
\end{tabular}

a. Eliminación por lista basada en todas las variables del procedimiento.

Tabla 9.

Estadísticos Total-Elemento
Se observa que la fiabilidad del instrumento es de .846, es decir, que la consistencia interna del instrumento es adecuada puesto que miden un mismo constructo y que están altamente correlacionados.

En la Tabla 9 se muestra la fiabilidad quitando el elemento donde se observa que la menor fiabilidad es de .831, reiterando que existe una adecuada consistencia interna, puesto que miden un mismo constructo y que están altamente correlacionados.

\begin{tabular}{ccccc}
\hline & $\begin{array}{c}\text { Media de la escala si } \\
\text { se elimina el elemento }\end{array}$ & $\begin{array}{c}\text { Varianza de la escala si } \\
\text { se elimina el elemento }\end{array}$ & $\begin{array}{c}\text { Correlación elemento- } \\
\text { total corregida }\end{array}$ & $\begin{array}{c}\text { Alfa de Cronbach si se } \\
\text { elimina el elemento }\end{array}$ \\
\hline Item1 & 45.40 & 65.187 & .531 & .834 \\
Item2 & 46.24 & 66.702 & .373 & .843 \\
Item5 & 45.95 & 63.859 & .465 & .838 \\
Item7 & 46.50 & 62.512 & .520 & .834 \\
Item10 & 46.11 & 63.451 & .520 & .834 \\
Item12 & 46.31 & 63.550 & .569 & .831 \\
Item13 & 46.42 & 66.746 & .488 & .837 \\
Item14 & 46.57 & 66.581 & .419 & .840 \\
Item16 & 46.54 & 65.584 & .483 & .837 \\
Item17 & 46.53 & 64.992 & .547 & .833 \\
Item18 & 46.41 & 67.134 & .392 & .841 \\
Item20 & 45.91 & 66.584 & .345 & .845 \\
Item21 & 46.14 & 65.953 & .470 & .837 \\
Item22 & 46.38 & 63.941 & .583 & .831 \\
Item25 & 45.91 & 65.177 & .460 & .838 \\
\hline
\end{tabular}

Teniendo en cuenta el estudio de Estrada, Batanero y Fortuny (2004) acerca de cómo se puede realizar la conformación de actitudes hacia la estadística, además de ser aceptada por muchos autores, siempre se presentará la controversia al respecto de cuáles deben ser las dimensiones que estructuran este constructo psicológico. Sin embargo, se puede considerar que las actitudes que los alumnos presentan hacia la estadística, pueden 
estar siendo influenciados por la manera en que ellos la aprenden y del cómo sienten su rendimiento en esta área.

La importancia de la estadística en la psicología, en especial el uso análisis factorial en el aporte de la elaboración de escalas, mostrando gran confiabilidad y validez, se puede ver en los trabajos de Woolfolk (2006) y Tejero y Casto (2011). Dado lo anterior, se realizó el análisis del instrumento de Validación de escala de actitudes hacia la estadística (Auzmendi 1992), con una muestra de 109 personas las cuales cursan actualmente estadística en el programa de psicología de la Universidad Piloto de Colombia. Se procedió con un análisis factorial y con los datos de este estudio, una primera conclusión es que el nuevo instrumento tendrá cinco dimensiones y 25 items, lo cual no es un instrumento válido en la actualidad para estudiantes de psicología de la Universidad Piloto de Colombia.

Se verificó la confiabilidad de las estimaciones, teniendo en cuenta el valor del Alfa de Cronbach (.831), cercano y adecuado a los presentados por Auzmendi, Alpha de chornbach de .90; Darías (2000) con Alpha de Cronbach de .90 y por Méndez y Marcía (2007), con un Alpha de Cronbach de .85 .

Según los datos arrojados, el instrumento se puede reducir a 4 factores con una fiabilidad de .831, lo que nos permite hacer un acercamiento a la teoría de Rosenberg y Hovland (1960) sobre los métodos de escalonamiento multidimensional donde se sugiere la dimensión cognitiva, la dimensión afectiva y la dimensión conductual. Por tanto, basado en esto, se realizan los factores de la siguiente manera: el primer factor es la dimensión cognitiva, importancia e interés de la estadística, y queda formado de la siguiente manera: 5 (La Estadística es demasiado teórica como para ser de utilidad práctica para el profesional medio), 10 (La Estadística puede ser útil para el que se dedique a la investigación pero no para el profesional medio) y 16 (Para el desarrollo profesional de nuestra carrera considero que existen otras asignaturas más importantes que la Estadística). Es la información que el individuo tiene sobre el objeto, lo que sabe o cree saber sobre él, su manera de representarlo y las categorías en las que lo incluye (Gagné, 1993); siendo un factor con mayor capacidad explicativa y fiabilidad con una varianza de $32.484 \%$.

El segundo factor es la dimensión conductual que hace referencia a la predisposición a desarrollar una determinada conducta, pudiendo interpretarse como un indicador de la esfera conductual (Kerlinger, 1988), tiene que ver con la predisposición activa y tendencia positiva hacia la estadística explicando así el 11.9\% de la varianza. Los ítems para el factor 2 quedan de la siguiente manera: 13 (Estoy calmado/a y tranquilo/a cuando me enfrento a un problema de Estadística), 17 (Trabajar con la Estadística hace que me sienta muy nervioso/a), 18 (No me altero cuando tengo que trabajar en problemas de Estadística) y 22 (La Estadística hace que me sienta incómodo/a y nervioso/a).

La dimensión afectiva se entiende como un continuo bipolar equidistante entre sentimientos negativos y positivos (Morales, 2002). Es decir, los sentimientos no se clasifican en sólo dos categorías (negativos o positivos), sino que se miden en una escala que es más negativa en un extremo y más positiva en el otro, en cuyo centro se halla la neutralidad; por tanto, 
se dividió en 2 factores: los sentimientos negativos y los sentimientos positivos (Feigl \& Scriven, 1967) hacia la estadística explican así el 9.99\% y el $8.919 \%$ respectivamente de la varianza.

Los ítems para el factor 3 son: 2 (La asignatura de Estadística me va bastante mal), 7 (La Estadística es una de las asignaturas que más temo), 12 (Cuando me enfrento a un problema de Estadística me siento incapaz de pensar con claridad) y 25 (La materia que se imparte en las clases de Estadística es muy poco interesante.); y para el Factor 4: 1 (Considero la estadística como una materia muy necesaria en la carrera de Psicología), 14 (La Estadística es agradable y estimulante para mí), 20 (Me provoca una gran satisfacción el llegar a resolver problemas de Estadística) y 21 (Para el desarrollo profesional de mi carrera una de las asignaturas más importantes que ha de estudiarse es la Estadística).

Lo que se observa a partir de los resultados frente a la Escala de Actitud Estadística es que no hay estabilidad factorial según la estructura dimensional propuesta por Auzmendi (1992), es decir, no es un instrumento adecuado en la actualidad para estudiantes de Psicología de segundo y tercer semestre de la Universidad Piloto de Colombia. Esto conllevó a utilizar un método de extracción y rotación distinto al empleado por Auzmendi (1992), lo que muestra que por el tiempo transcurrido podría cambiar los métodos de enseñanza y aprendizaje; en consecuencia, se disminuyen así los ítems según las condiciones actuales de la actitud hacia la estadística. Se observa que la varianza explicada fue $63.29 \%$, que es superior a estudios similares realizados bajo la misma escala como Auzmendi (1992), la cual fue de $60.70 \%$ y Darías (2000) que fue de $60.70 \%$ e inferior pero muy cercana a Méndez y Macía (2007) con $65.57 \%$ de la varianza explicada.

Por tanto, se realizó una exigente selección de ítems de la Escala de Actitudes Estadísticas, hasta alcanzar un instrumento actual, válido y fiable, con dimensiones unipolares, consistentes y robustas. En este sentido, se propone un instrumento de quince ítems y cuatro dimensiones, justificado no sólo psicométricamente sino también desde una perspectiva teórica y de congruencia de significado o contenido. Este nuevo instrumento se evaluó mediante la Medida de Adecuación de la Muestra KMO propuesta por Kaiser, Meyer \& Olkin (1997), donde se evidencia que con un (KMO) $=.784$, los datos se pueden trabajar por análisis factorial, dado que las correlaciones entre las 15 variables pueden ser explicadas por otras variables. Se obtuvo así una fiabilidad del instrumento final de .846 , es decir, que la consistencia interna del instrumento es adecuada puesto que miden un mismo constructo y que están altamente correlacionados, lo que demuestra su validez psicométrica.

Respecto a la validez, en cuanto a la congruencia de significado o contenido, se hizo un acercamiento a la teoría de Rosenberg y Hovland (1960) sobre los métodos de escalonamiento multidimensional donde se sugiere la dimensión cognitiva, la dimensión afectiva (sentimientos positivos - sentimientos negativos) y la dimensión conductual, teniendo como resultado a la dimensión cognitiva como el factor más importante con una varianza explicada de $32.48 \%$, lo cual nos indica que la percepción de los estudiantes de psicología es que la estadística no es 
una asignatura de importancia para ellos. Además, dado el peso tan alto respecto a la varianza total explicada del factor cognitivo, si se logra reducir la dificultad que perciben o si se enfocan los cursos buscando una mejora en la propia capacidad cognitiva respecto a la estadística, se podrá conseguir un aumento en la afectividad hacia la Estadística y, por ende, una mejora en el rendimiento académico de los estudiantes (Blanco, 2004).

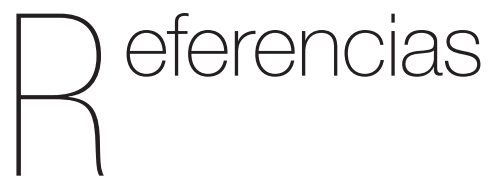

Auzmendi, E. (1992). Las actitudes hacia la matemática-estadística en las enseñanzas medias y universitarias. Bilbao: Mensajero.

Aliaga, J., \& Pecho, J. (2000). Evaluación de la actitud hacia la matemática en estudiantes secundarios. Paradigmas, 1(2), 1-2.

Bazán, J. L., \& Aparicio, A. S. (2006). Las actitudes hacia la matemática estadística dentro de un modelo de aprendizaje. Revista de Educación de la Pontificia Universidad Católica, 15, 7-20.

Blanco, A. (2004). Enseñar y aprender estadística en las titulaciones universitarias de ciencias sociales: apuntes sobre el problema desde una perspectiva pedagógica. Madrid: Universidad Pontificia Comillas.

Cazau, P. (2003). Vocabulario de Psicología. Recuperado el 12 enero de 2016 de www. redpsicologia.com
Clemente, M. (1992). Psicología Social: Métodos y Técnicas de Investigación. Madrid: Eudema.

Cronbach, L. (1951). Coefficient alpha and the internal structure of tests. Psychometrika, 16, 297-334.

Darías, E. J. (2000). Escala de actitudes hacia la estadística. Psicothema 12(2), 175-178.

Eagly, A., \& Chaiken, S. (1993). The psychology of attitudes. Reviewed by Christopher Leone, University of North Florida, 15, 459-466.

Estrada, A., Batanero, C., \& Fortuny, J. M. (2004). Un estudio comparado de las actitudes hacia la estadística en profesores en formación y en ejercicio. Enseñanza de las Ciencias, 22(2), 263-274.

Feigl, H., \& Scriven, M. (1967). LoS fundamentos de la ciencia y los conceptos de la psicología y del psicoanálisis. Chile: Universidad de Chile.

Gagné, R. (1993). Las condiciones del aprendizaje. México, D.F: McGraw-Hill.

García, E., Gil, J., \& Rodríguez, G. (2000). Análisis Factorial. Cuadernos de Estadística. Madrid: Editorial La Muralla.

Gil, J. (1999). Actitudes hacia la estadística. Incidencia de las variables sexo y formación previa. Revista Española de Pedagogía, 214, 567-590.

Gorsuch, R. (1983). Factor Analysis. Madrid: Edition. LEA

Hair, J., Black, W., \& Babin, B. (2009). Multivariate Data Analysis (7th Edition). USA: Pearson 
Kaiser, Meyer, \& Olkin (1977). A study of a measure of sampling adequacy for factor-analytic correlation matrices. 12 enero de 2016, de Multivariate Behavioral Research Sitio web: http: analisis_ datosyMultivariable/20factor_SPSS.pdf

Kerlinger, F. (1988). Investigación del comportamiento. México, D.F.: McGraw-Hill.

Méndez, D., \& Macia, F. (2007). Análisis factorial confirmatorio de la escala de actitudes hacia la estadística. Cuadernos de Neuropsicología 1(3), 174 -371.

Ministerio de Nacional de Educación. (2006). Estándares básicos de competencias en lenguaje, matemáticas, ciencias y ciudadanía. Primera edición mayo de 2006.
Morales, J. (2002). Psicología Social. Buenos Aires: Pearson.

Rosenberg, M., \& Hovland, C. (1960). Cognitive, affective and behavioral components of attitudes. In C.I. Hovland and M.J. Rosenberg (Eds.). Attitude Organization and change (pp. 1-14). New Haven: Yale University Press.

Tejero, C., \& Casto, M. (2011). Validación de la escala de actitudes hacia la estadística en estudiantes españoles de ciencias de la actividad física y del deporte. Revista Colombiana de Estadística, 34(1), 1-14.

Woolfolk, A. (2006). Psicología Educativa (Novena edición). Madrid: Pearson Educación. 